\title{
Análisis de la producción de publicaciones científicas en inteligencia artificial aplicada a la formulación de políticas públicas
}

\author{
Analysis of the production of scientific publications in artificial intelligence applied to \\ the formulation of public policies
}

\section{Análise da produção de publicações científicas sobre inteligência artificial aplicada à formulação de políticas públicas}

\author{
Juan Manuel Sánchez-Céspedes ${ }^{1}$ \\ Juan Pablo Rodríguez-Miranda ${ }^{2}$ \\ Octavio José Salcedo-Parra ${ }^{3}$ (1)
}

Recibido: marzo 2020

Aceptado: agosto 2020

Para citar este artículo: Sánchez-Céspedes, D. M., Rodríguez-Miranda, J. P., Salcedo-Parra, O. J. (2020). Análisis de la producción de publicaciones científicas en inteligencia artificial aplicada a la formulación de políticas públicas. Revista Científica, 39(3), 353-368. https://doi.org/10.14483/23448350.16301

\section{Resumen}

El objetivo de este artículo es analizar las publicaciones científicas especializadas en el uso de herramientas de inteligencia artificial en el proceso de toma de decisiones durante la formulación de políticas públicas. Como herramienta metodológica se creó una ecuación de búsqueda para ubicar las publicaciones concernientes, la cual fue probada y perfeccionada varias veces para mejorar los resultados encontrados. Esta ecuación fue aplicada en la base de datos de Scopus, con lo cual se obtuvieron 1154 publicaciones, a las que se aplicaron indicadores bibliométricos. En los resultados obtenidos se encontró que principalmente esta área ha tenido un gran crecimiento en la última década; cuyos países con mayor producción son: Estados Unidos, República Popular de China y el Reino Unido. Al comparar producción científica con las entidades patrocinantes se pudo concluir la importancia del apoyo gubernamental para desarrollo científico de un país.

Palabras clave: inteligencia artificial, política pública, toma de decisiones, formulación de políticas, análisis bibliométrico.

\begin{abstract}
The objective of this article is to analyze the scientific publications made regarding the use of artificial intelligence tools in the decision-making process in the formulation of public policies. The methodology used was initially to create a search equation to locate the publications in this regard, which was tested and refined several times to improve the results found, this equation was applied in
\end{abstract}

1. Universidad Distrital Francisco José de Caldas, Bogotá, Colombia. jmsanchezc@udistrital.edu.co

2. Universidad Distrital Francisco José de Caldas, Bogotá, Colombia. jprodriguezm@udistrital.edu.co

3. Universidad Distrital Francisco José de Caldas, Bogotá, Colombia. osalcedo@udistrital.edu.co 
the SCOPUS database, with which 1.154 publications were obtained, to which bibliometric indicators were applied. The main results obtained were that this area has had great growth in the last decade, where the countries with the highest production are the United States, the People's Republic of China and the United Kingdom. By comparing scientific production with financing entities, it was possible to conclude the importance of government support for scientific development in a country.

Keywords: artificial intelligence, public policy, decision-making, policy formulation, bibliometric analysis.

\section{Resumo}

O objetivo deste artigo é analisar as publicações científicas realizadas sobre o uso de ferramentas de inteligência artificial no processo de tomada de decisão na formulação de políticas públicas. A metodologia utilizada foi inicialmente criar uma equação de busca para localizar as publicações a esse respeito, que foi testada e refinada várias vezes para melhorar os resultados encontrados, esta equação foi aplicada no banco de dados SCOPUS, com o qual foram obtidas 1.154 publicações, aos quais indicadores bibliométricos foram aplicados. Os principais resultados obtidos foram que essa área teve um grande crescimento na última década, onde os países com maior produção são Estados Unidos, República Popular da China e Reino Unido. Ao comparar a produção científica com as entidades financiadoras, foi possível concluir a importância do apoio do governo ao desenvolvimento científico em um país.

Palavras-chaves: inteligência artificial, políticas públicas, tomada de decisão, formulação de políticas, análise bibliométrica.

\section{Introducción}

El termino de inteligencia artificial (IA) se usó por primera vez en 1958 por los científicos McCarthy, Minsky, Rocherster y Shannon, quienes la definieron como "la ciencia y la ingeniería para crear máquinas inteligentes" (Poole y Mackworth, 2017). Una conceptualización más reciente la define como el área de la ciencia de la computación que tiene como objetivo emular las funciones cognitivas del ser humano mediante el uso de sistemas computacionales (Manyika et al., 2017). Una explicación más amplia del concepto la presentan Poole y Mackworth (2017), quienes definen la IA como "el estudio y diseño de agentes inteligentes"; entendiendo que un agente es "algo" que actúa en un entorno que se denomina "inteligente" cuando realiza acciones de acuerdo con las circunstancias y los objetivos propuestos (Russell y Norvig, 2009). Se han desarrollado diversas técnicas de IA, algunas de estas son: redes neuronales, lógica difusa, algoritmos genéticos, modelos basados en agentes, entre otras más. Estas herramientas se han usado para dar solución a diferentes clases de problemas, uno de ellos ha sido ayudar al ser humano a mejorar el proceso de toma de decisiones, teniendo en cuenta la racionalidad limitada propia de las personas, la cual explica que el ser humano toma decisiones de forma parcialmente irracional debido a las limitaciones cognitivas, de información y de tiempo (Simon, 1979).

La IA ha ayudado al proceso de toma de decisiones en diferentes áreas del conocimiento. A continuación, se nombran algunas de estas. La inteligencia de negocios que ayuda al proceso de toma de decisiones gerenciales (Chávez et al., 2018; Flath y Stein, 2018; Lin et al., 2017; Marine-Roig, 2017; Zamora, 2017). También, en el área de la salud para mejorar los procesos de toma de decisiones en atención médica (Chen et al., 2017; Chen et al., 2020; Jiang et al., 2017; Mehta y Pandit, 2018; Ramesh et al., 2016; Thesmar et al., 2019). En el mercado de valores para determinar en qué momento comprar o vender acciones (Chatzis et al., 2018; Chen et al., 2018; Fernández et al., 2013; Nayak et al., 2016; Shah et al., 2018). En el sector bancario para la aprobación de créditos (Okesola et al., 2018; Peña et al., 2017; Turkson et al., 2016). También se ha usado la IA para procesos de selección de personal (Menon y Rahulnath, 2017; Nawaz, 2019; Van Esch et al., 2019). Otra aplicación ha sido en generar sistemas de alertas tempranas para eventos meteorológicos 
(Moon et al., 2019; Moreno et al., 2018; Santacreu et al., 2015; Šaur, 2017). Como las ya mencionadas, hay muchas más aplicaciones en la cuales se han usado estas herramientas tecnológicas. Por lo cual, la IA ha comprobado que son instrumentos muy útiles para el proceso de toma de decisiones.

Una de las áreas que tiene grandes inconvenientes en el proceso de toma de decisiones es la formulación de políticas públicas, esto debido al alto grado de complejidad. Esta se puede explicar a través del modelo de caneca de basura, el cual revela que el proceso de toma de decisiones se hace sobre una anarquía organizada. Esto debido a que todos los elementos que intervienen en el proceso de toma de decisiones como los problemas, las soluciones, los participantes o las oportunidades de elección se entremezclan entre sí de forma caótica y al mismo tiempo como si estuvieran dentro de una caneca de basura (Cohen et al., 1972); haciendo que el proceso sea complejo y provoque que no siempre se tome la decisión más idónea. Un ejemplo en el cual se evidencia que no se han tomado las mejores decisiones en política pública es el caso colombiano, pues las políticas públicas agrarias implementadas han tenido efectos contrarios a los esperados, los cuales han perjudicado a la población campesina y profundizado la inequidad social (Baudasse y Calderón, 2009; Gómez, 2016; Morales et al., 2017; Ospina, 2017; Soto, 2003; Vargas et al., 2016).

Por estos motivos, el presente artículo tiene como objetivo hacer una revisión de las publicaciones científicas relacionadas al uso de herramientas de IA en el proceso de toma de decisiones al realizar la formulación de políticas públicas. Para lo cual el artículo se divide en la presente introducción, sigue con la metodología aplicada, luego de ello se presenta los resultados obtenidos y se finaliza con las conclusiones.

\section{Metodología}

La metodología aplicada fue de tipo descriptivo con enfoque cuantitativo. El primer paso consistió en desarrollar la ecuación de búsqueda y realizar la respectiva prueba en el repositorio de publicaciones científicas de Scopus. Este proceso de generación de la ecuación fue de carácter iterativo; es decir, se observan los resultados obtenidos con la ecuación y se va mejorando para captar mejores resultados. Luego, se aplicaron los indicadores bibliométricos; ya con estos se analizaron y se obtuvieron las conclusiones. A continuación, se explica en mayor detalle cada etapa de la metodología.

\section{Construcción de ecuación de búsqueda}

Para la construcción de la ecuación de búsqueda se tomó como base los temas de la investigación: IA, política pública y toma de decisiones. Con los temas se buscaron las palabras claves que los autores y las publicaciones utilizaban para referenciar el tema. Como se explicó, la ecuación se probó sobre la base de datos, se analizaron los resultados obtenidos y se fue mejorando la ecuación, para así optimizar los resultados. Al final se obtuvo la ecuación, con la cual se captaron 1.154 publicaciones. La ecuación de búsqueda se presenta a continuación.

TITLE-ABS-KEY ("artificial intelligence" OR "Cybernetics" OR "Neural Network" OR "Fuzzy logic" OR "Machine learning" OR "Data Mining" OR "Expert systems" OR "genetic algorithms" OR "neuro-fuzzy" OR "agent-based model" OR "particle swarm" OR "cellular automata" OR "Ant Colony Optimization" OR "Big Data" OR "Bayesian Network") AND TITLE-ABS-KEY ("public poli*" OR "National poli*" OR "Government poli*" OR "political decision-mak" OR "policy mak*") AND TITLEABS-KEY ("decision mak*" OR "Decision Supp*")

\section{Aplicación de indicadores bibliométricos}

A los datos bibliográficos de las 1.154 publicaciones que resultaron del proceso de búsqueda se aplicaron los siguientes indicadores bibliométricos. 
- Histórico de publicaciones: este indicador muestra el número de publicaciones realizadas por año.

- Autores de publicaciones: muestra el listado de autores y el número de publicaciones científicas de cada uno.

- País de origen: presenta el número de publicaciones por país, acorde al país donde se realizó la publicación.

- Patrocinadores: indica quiénes financiaron las investigaciones que generaron las publicaciones. El número corresponde a las publicaciones que tiene asociada cada patrocinador.

- Palabras claves: para analizar y agrupar las palabras claves se usó el método de fuerza de asociación. Este método busca determinar cuáles categorías se relacionan más entre ellas y se agrupan acorde a ello. Para lo cual se utilizó el software libre VosViewer, el cual funciona para el análisis bibliométrico pues ayuda a crear redes bibliométricas para ver gráficamente estas relaciones.

\section{Análisis de resultados y conclusiones}

A partir de los resultados obtenidos con los indicadores bibliométricos, se analizaron y se obtuvieron las conclusiones.

\section{Resultados}

Los datos se analizaron desde cinco perspectivas: histórico de publicaciones, autores, países, patrocinadores y palabras claves.

\section{Histórico de publicaciones}

En la figura 1 se presentan las publicaciones realizadas entre los años 1991 hasta el 2020. En el 2020 solo corresponde al primer trimestre, por eso no se debe comparar la tendencia con los otros años.

Como se observa en la figura 1, entre el año 2004 al 2008 la tendencia de publicaciones estaba estable; pero, a partir del año 2009 se presentó un crecimiento exponencial de las publicaciones

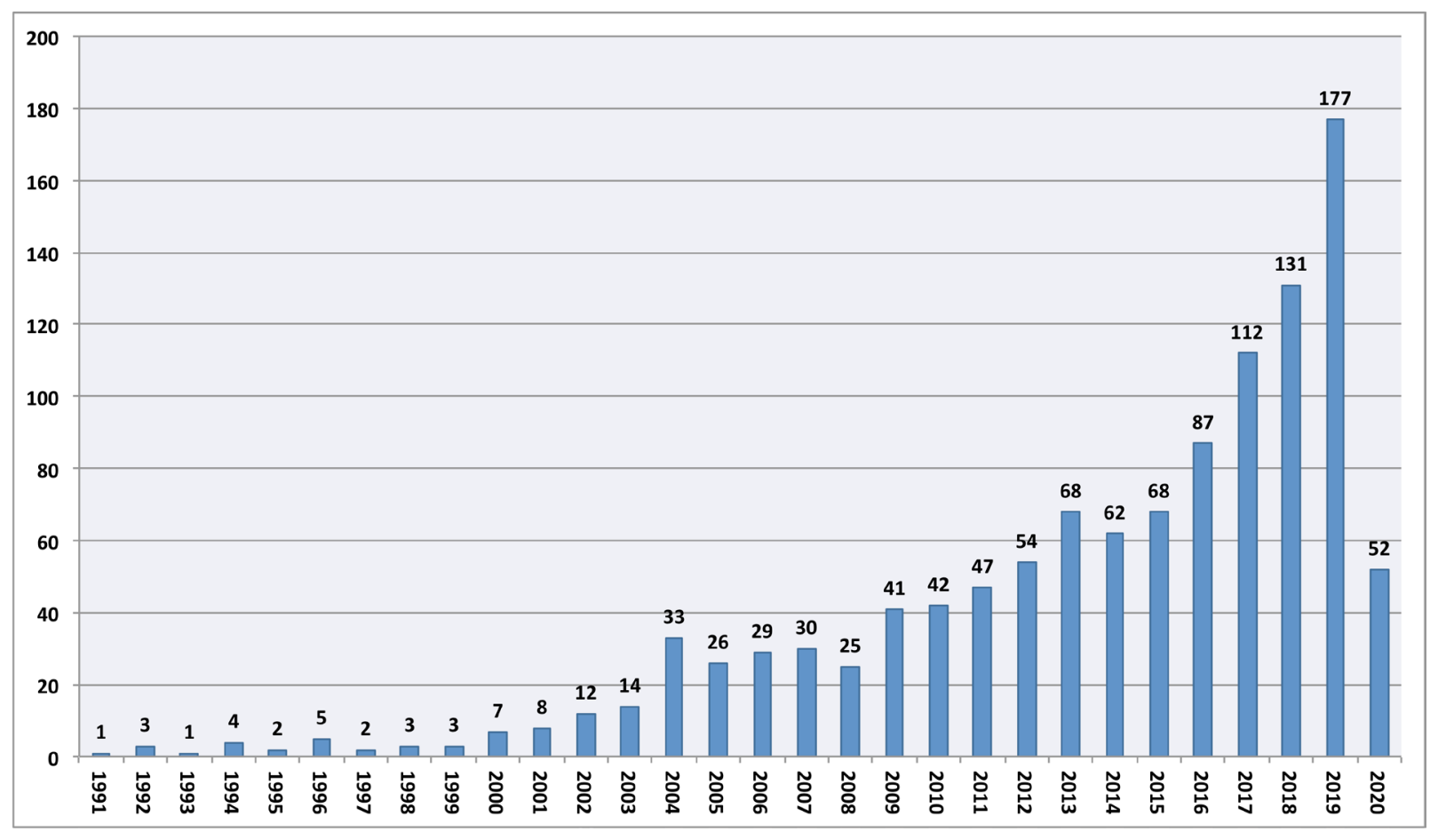

Figura 1. Histórico de publicaciones.

Fuente: adaptado por los autores de Scopus. 
que se ha mantenido hasta el 2019, lo cual evidencia el crecimiento del interés científico sobre este tema durante la última década.

\section{Principales autores}

En la figura 2 se presenta el listado de los principales autores sobre el tema, los cuales tienen por lo menos cuatro publicaciones en el área.

El investigador que más tiene publicaciones es Yannis Charalabidis, profesor del área de Gobierno digital y de formulación de políticas públicas. Él trabaja en la Universidad Aegean School of Sciences, en el Departamento de Información e Ingeniería en Sistemas de Comunicaciones. Charalabidis ha trabajado en conjunto con Evripidis Loukis en cinco publicaciones de las seis que tiene Loukis; Charalabidis también ha trabajado con Aggeliki Androutsopoulou en cuatro publicaciones. Loukis y Androutsopoulou son también profesores de The Aegean School of Sciencesen en Grecia. En la tabla 1 se presenta las publicaciones de Charalabidis, Loukis y Androutsopoulou en el área.

Charalabidis, Loukis y Androutsopoulou han desarrollado investigaciones enfocadas en el uso de herramientas TIC que ayuden a la formulación de políticas públicas. Por lo cual al inicio realizaron investigaciones de cómo usar estas herramientas para la recopilación de información en las redes sociales y así facilitar el proceso de formulación de políticas públicas (Androutsopoulou et al., 2016; Charalabidis y Loukis, 2011; Charalabidis et al., 2011; Charalabidis et al., 2013). Luego se enfocaron al uso de herramientas como big data y minería de datos para recopilar y procesar información que ayudase a la construcción de políticas públicas (Androutsopoulou y Charalabidis, 2018; Androutsopoulou et al., 2018). El último trabajo publicado con respecto al tema corresponde a una

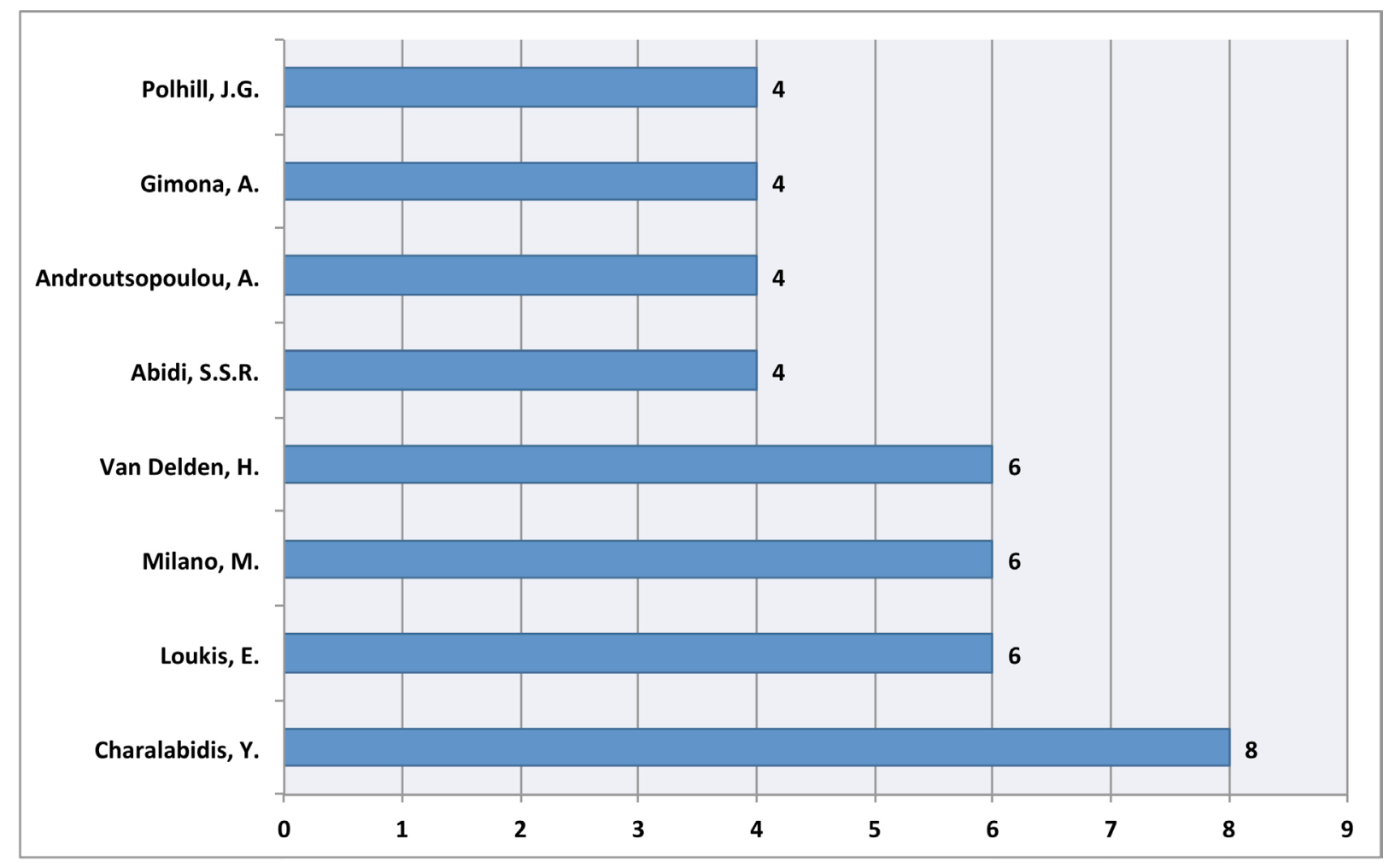

Figura 2. Principales autores.

Fuente: adaptado por los autores de Scopus. 
Tabla 1. Publicaciones de Charalabidis, Loukis y Androutsopoulou

\begin{tabular}{|c|c|c|c|c|}
\hline Autores & Título del documento & año & Fuente del documento & $\begin{array}{c}\text { Tipo de } \\
\text { documento }\end{array}$ \\
\hline Loukis, Maragouda y Kyriakou & $\begin{array}{l}\text { Economic Crisis Policy Analytics } \\
\text { Based on Artificial Intelligence }\end{array}$ & 2019 & $\begin{array}{l}\text { Lecture Notes in Computer } \\
\text { Science (including subseries } \\
\text { Lecture Notes in Artificial } \\
\text { Intelligence and Lecture } \\
\text { Notes in Bioinformatics) }\end{array}$ & $\begin{array}{l}\text { Conference } \\
\text { Paper }\end{array}$ \\
\hline $\begin{array}{l}\text { Androutsopoulou, } \\
\text { Karacapilidis, Loukis y } \\
\text { Charalabidis }\end{array}$ & $\begin{array}{l}\text { Combining Technocrats" Expertise } \\
\text { with Public Opinion through an } \\
\text { Innovative e-Participation platform }\end{array}$ & 2018 & $\begin{array}{l}\text { IEEE Transactions on } \\
\text { Emerging Topics in } \\
\text { Computing }\end{array}$ & Article in Press \\
\hline $\begin{array}{l}\text { Androutsopoulou y } \\
\text { Charalabidis }\end{array}$ & $\begin{array}{l}\text { A framework for evidence based } \\
\text { policy making combining big data, } \\
\text { dynamic modelling and machine } \\
\text { intelligence }\end{array}$ & 2018 & $\begin{array}{l}\text { ACM International } \\
\text { Conference Proceeding } \\
\text { Series }\end{array}$ & $\begin{array}{l}\text { Conference } \\
\text { Paper }\end{array}$ \\
\hline $\begin{array}{l}\text { Androutsopoulou, Mureddu, } \\
\text { Loukis y Charalabidis }\end{array}$ & $\begin{array}{l}\text { Passive expert-sourcing for policy } \\
\text { making in the European union }\end{array}$ & 2016 & $\begin{array}{l}\text { Lecture Notes in Computer } \\
\text { Science (including subseries } \\
\text { Lecture Notes in Artificial } \\
\text { Intelligence and Lecture } \\
\text { Notes in Bioinformatics) }\end{array}$ & $\begin{array}{l}\text { Conference } \\
\text { Paper }\end{array}$ \\
\hline Spiliotopoulou y Charalabidis & $\begin{array}{l}\text { Web } 2.0 \text { in governance: A } \\
\text { framework for utilizing social } \\
\text { media and opinion mining } \\
\text { methods and tools in policy } \\
\text { deliberation }\end{array}$ & 2015 & $\begin{array}{l}\text { Mobile Computing and } \\
\text { Wireless Networks: } \\
\text { Concepts, Methodologies, } \\
\text { Tools, and Applications }\end{array}$ & Chapter Book \\
\hline Spiliotopoulou y Charalabidis & $\begin{array}{l}\text { Web } 2.0 \text { in governance: A } \\
\text { framework for utilizing social } \\
\text { media and opinion mining } \\
\text { methods and tools in policy } \\
\text { deliberation }\end{array}$ & 2015 & $\begin{array}{l}\text { Social Media and } \\
\text { Networking: Concepts, } \\
\text { Methodologies, Tools, and } \\
\text { Applications }\end{array}$ & Chapter Book \\
\hline $\begin{array}{l}\text { Charalabidis, Loukis, } \\
\text { Spiliotopoulou y } \\
\text { Diamantopoulou }\end{array}$ & $\begin{array}{l}\text { A framework for utilizing Web } \\
2.0 \text { social media for participative } \\
\text { governance }\end{array}$ & 2013 & $\begin{array}{l}\text { Proceedings of the European, } \\
\text { Mediterranean and Middle } \\
\text { Eastern Conference on } \\
\text { Information Systems, EMCIS } \\
2013\end{array}$ & $\begin{array}{l}\text { Conference } \\
\text { Paper }\end{array}$ \\
\hline $\begin{array}{l}\text { Charalabidis, Loukis y } \\
\text { Androutsopoulou }\end{array}$ & $\begin{array}{l}\text { Enhancing participative policy } \\
\text { making through modelling and } \\
\text { simulation: A state of the art review }\end{array}$ & 2011 & $\begin{array}{l}\text { Proceedings of the European, } \\
\text { Mediterranean and Middle } \\
\text { Eastern Conference on } \\
\text { Information Systems - } \\
\text { Informing Responsible } \\
\text { Management: Sustainability } \\
\text { in Emerging Economies, } \\
\text { EMCIS } 2011\end{array}$ & $\begin{array}{l}\text { Conference } \\
\text { Paper }\end{array}$ \\
\hline Charalabidis y Loukis & $\begin{array}{l}\text { Transforming government } \\
\text { agencies" approach to } \\
\text { eparticipation through efficient } \\
\text { exploitation of social media }\end{array}$ & 2011 & $\begin{array}{l}\text { 19th European Conference } \\
\text { on Information Systems, ECIS } \\
2011\end{array}$ & $\begin{array}{l}\text { Conference } \\
\text { Paper }\end{array}$ \\
\hline
\end{tabular}

Fuente: adaptado por los autores de Scopus.

metodología para el uso de big data en la formulación de políticas públicas en crisis económicas (Loukis et al., 2019).

Michela Milano es profesora en el Departamento de Ciencias de la Computación e Ingeniería de la Universidad de Bolonia. Es vicepresidenta de la
Asociación Europea de Inteligencia Artificial (EurAl) y miembro del Comité Ejecutivo de la Asociación para la Programación de Restricciones y de la Asociación Italiana de Inteligencia Artificial. En la tabla 2 se presentan las publicaciones de Milano con respecto al tema. 
Tabla 2. Publicaciones de Michela Milano

\begin{tabular}{lcll}
\hline \multicolumn{1}{c}{\begin{tabular}{c}
\multicolumn{1}{c}{ Título de documento } \\
$\begin{array}{l}\text { Merging observed and self-reported } \\
\text { behaviour in agent-based simulation: A } \\
\text { case study on photovoltaic adoption }\end{array}$
\end{tabular}} & Año & \multicolumn{1}{c}{ Fuente del documento } & $\begin{array}{c}\text { Tipo de } \\
\text { documento }\end{array}$ \\
$\begin{array}{l}\text { Sustainable policy making: A strategic } \\
\text { challenge for artificial intelligence }\end{array}$ & 2019 & Applied Sciences (Switzerland) & Article \\
$\begin{array}{l}\text { Multi-criteria optimal planning for } \\
\text { energy policies in CLP? }\end{array}$ & 2014 & Al Magazine & Article \\
$\begin{array}{l}\text { Optimization for policy making: The } \\
\text { cornerstone for an integrated approach }\end{array}$ & 2013 & $\begin{array}{l}\text { Lecture Notes in Computer Science (including } \\
\text { subseries Lecture Notes in Artificial Intelligence } \\
\text { and Lecture Notes in Bioinformatics) }\end{array}$ & $\begin{array}{c}\text { Conference } \\
\text { Paper }\end{array}$ \\
$\begin{array}{l}\text { Sustainable energy policies: Research } \\
\text { challenges and opportunities }\end{array}$ & 2013 & $\begin{array}{l}\text { Proceedings -Design, Automation and Test in } \\
\text { Europe (Date) }\end{array}$ & $\begin{array}{l}\text { Conference } \\
\text { Paper }\end{array}$ \\
$\begin{array}{l}\text { Constraint and optimization techniques } \\
\text { for supporting policy making }\end{array}$ & 2012 & $\begin{array}{l}\text { International Symposium on Artificial } \\
\text { Intelligence and Mathematics, Isaim 2012 }\end{array}$ & $\begin{array}{l}\text { Conference } \\
\text { Paper }\end{array}$ \\
\hline
\end{tabular}

Fuente: adaptado por los autores de Scopus.

Las investigaciones de Michela Milano sobre el tema se han enfocado en cómo usar herramientas de IA, en especial modelos basados en agentes para que ayuden a determinar los posibles resultados que se puedan presentar al implementar las políticas públicas. Para ello Milano ha desarrollado sistemas de soporte de decisiones (DSS-Decision Support System) muy útiles para los formuladores de política pública (Gavanelli et al., 2014; Milano, 2013a). Principalmente, se ha enfocado en aplicaciones sobre el sector energético (Borghesi y Milano, 2019; Gavanelli et al., 2016; Milano, 2013b) y evaluación ambiental (Milano et al., 2014).

Hedwig Van Delden es una de las pioneras en el tema a nivel mundial. Actualmente es la directora del Research Institute for Knowledge Systems (RIKS) en Holanda y es profesora asociada de la Universidad de Adelaide. En la tabla 3 se presentan las publicaciones de Van Delden sobre el tema.

Las publicaciones de Van Delden tratan sobre desarrollos de DSS, los cuales ayudan a soportar el proceso de toma decisiones en formulación de políticas públicas en la Unión Europea (Huser et al., 2009; Van Delden, 2009; Van Delden y Hurkens, 2011; Van Delden et al., 2009; Van Delden et al., 2011; Van Delden et al., 2010). Estos sistemas fueron desarrollados en el Research Institute for Knowledge Systems (RIKS).

Syed Sibte Raza Abidi es profesor de informática y director de informática sanitaria en la Facultad de Informática de la Universidad de Dalhousie (Halifax, Canadá). Realiza investigaciones en las áreas de informática sanitaria, gestión del conocimiento, web semántica y análisis de datos, para lo cual ha usado herramientas como minería de datos o modelamiento basados en agentes para descubrir información que ayude a los formuladores de políticas públicas, en especial en áreas de la salud en formular políticas (Sibte y Abidi, 2001; Zaidi et al., 2002; Zaidi et al., 2004). En la tabla 4 se presenta las publicaciones sobre el tema.

Gary Polhill es investigador senior del James Hutton Institute en el Reino Unido, se graduó en IA y es doctor en redes neuronales; actualmente es el presidente del European Social Simulation Association (Essa). Alessandro Gimona también es un investigador del James Hutton Institute, él es un ecólogo espacial y un geógrafo con experiencia en sistemas terrestres y acuáticos, con magister y doctorado en el área. Polhill y Gimona han trabajado en conjunto y tienen cuatro publicaciones en el tema. En la tabla 5 se presentan estas publicaciones. 
Tabla 3. Publicaciones de Hedwig Van Delden

\section{Título del documento}

A generic integrated spatial decision support system for urban and regional planning

A methodology for the design and development of integrated models for policy support

Integrated assessment of agricultural policies with dynamic land use change modelling

Development of an integrated spatial decision support system (ISDSS) for local government in New Zealand

Towards a modelling framework for integrated assessment in arid and semiarid regions

Integration of socio-economic and biophysical models to support sustainable development

\section{Fuente del documento}

MODSIM 2011 - 19th International Congress on

2011 Modelling and Simulation - Sustaining Our Future:

Understanding and Living with Uncertainty

2011 Environmental Modelling and Software

Article

2010 Ecological Modelling

Article

18th World IMACS Congress and MODSIM09

International Congress on Modelling and Simulation: Conference Interfacing Modelling and Simulation with Mathematical Paper and Computational Sciences, Proceedings

18th World IMACS Congress and MODSIM09

2009 International Congress on Modelling and Simulation: Conference Interfacing Modelling and Simulation with Mathematical Paper and Computational Sciences, Proceedings

18th World IMACS Congress and MODSIM09

2009 International Congress on Modelling and Simulation: Conference Interfacing Modelling and Simulation with Mathematical Paper and Computational Sciences, Proceedings

Fuente: adaptado por los autores de Scopus.

Tabla 4. Publicaciones de Syed Sibte Raza Abidi

\begin{tabular}{|c|c|c|c|}
\hline Título del documento & Año & Fuente del documento & Tipo de documento \\
\hline $\begin{array}{l}\text { ADMI: A multi-agent architecture to autonomously } \\
\text { generate data mining services }\end{array}$ & 2004 & $\begin{array}{l}2004 \text { 2nd International IEEE } \\
\text { Conference "Intelligent } \\
\text { Systems" - Proceedings }\end{array}$ & $\begin{array}{l}\text { Int. IEEE Conf. Intell. Syst. } \\
\text { Proc. }\end{array}$ \\
\hline $\begin{array}{l}\text { Leveraging intelligent agents for knowledge discovery } \\
\text { from heterogeneous healthcare data repositories }\end{array}$ & 2002 & $\begin{array}{l}\text { Studies in Health Technology } \\
\text { and Informatics }\end{array}$ & Chapter book \\
\hline $\begin{array}{l}\text { Knowledge management in healthcare: Towards } \\
\text { "knowledge-driven" decision-support services }\end{array}$ & 2001 & $\begin{array}{l}\text { International Journal of } \\
\text { Medical Informatics }\end{array}$ & Int. J. Med. Inform. \\
\hline $\begin{array}{l}\text { Applying data mining in healthcare: An info-structure } \\
\text { for delivering "data-driven" strategic services }\end{array}$ & 1999 & $\begin{array}{l}\text { Studies in Health Technology } \\
\text { and Informatics }\end{array}$ & Chapter book \\
\hline
\end{tabular}

Fuente: adaptado por los autores de Scopus.

Tabla 5. Publicaciones de Gimona y Polhill

\begin{tabular}{|c|c|c|c|}
\hline Título & Año & Fuente del Documento & $\begin{array}{c}\text { Tipo de } \\
\text { Documento }\end{array}$ \\
\hline $\begin{array}{l}\text { Nonlinearities in biodiversity incentive schemes: } \\
\text { A study using an integrated agent-based and } \\
\text { metacommunity model }\end{array}$ & 2013 & Environmental Modelling and Software & Article \\
\hline $\begin{array}{l}\text { Exploring robustness of biodiversity policy with a } \\
\text { coupled meta community and agent-based model }\end{array}$ & 2011 & Journal of Land Use Science & Article \\
\hline Sinks, sustainability, and conservation incentives & 2011 & Sources, Sinks and Sustainability & Book Chapter \\
\hline $\begin{array}{l}\text { Analysis of incentive schemes for biodiversity } \\
\text { using a coupled agent-based model of land use } \\
\text { change and species metacommunity model }\end{array}$ & 2010 & $\begin{array}{l}\text { Modelling for Environment's Sake: } \\
\text { Proceedings of the 5th Biennial Conference } \\
\text { of the International Environmental Modelling } \\
\text { and Software Society, iEMSs } 2010\end{array}$ & Conference Paper \\
\hline
\end{tabular}

Fuente: adaptado por los autores de Scopus. 
Estas publicaciones son resultado de una investigación en el cual buscaban determinar cómo a través de subsidios gubernamentales se lograba el mejor uso de la tierra para el sector agrícola en Escocia (Reino Unido), siendo ser más eficientes en su producción e integrando agricultura sostenible para de esta forma disminuir el impacto ambiental negativo. La herramienta de IA que usaron fue el modelo basado en agentes (Gimona y PolhiII, 2011; Gimona et al., 2011; Polhill et al., 2010, 2013).

Al observar las publicaciones de los principales investigadores en el tema se encuentra que se han preocupado en desarrollar DSS que ayuden al proceso de formulación de políticas públicas, donde las herramientas de IA más utilizadas son minería de datos y modelos basados en agentes.

\section{Publicaciones por países}

En la figura 3 se presenta los países líderes en investigación sobre formulación de políticas públicas usando IA.
Como se observa en la figura 3, el líder en publicaciones sobre el uso de IA para la formulación de políticas públicas es Estados Unidos, en un segundo grupo se encuentra China y el Reino Unido. Después se encuentra Italia y luego otros países que en su mayoría están ubicados en Asia y Europa. Al identificar cuáles son los países que más publican sobre el tema se observa que la mayoría de estos son países desarrollados y se encuentran ubicados en Asia, Europa y Norte América; el único país latinoamericano que se encuentra en este grupo es Brasil con 28 publicaciones, lo que realmente es muy poco comparado con Estados Unidos, China o Reino Unido.

\section{Patrocinadores}

En la figura 4 se presentan los principales patrocinadores de las investigaciones sobre el tema.

Se observa en la figura 4 que las tres principales organizaciones que apoyan las investigaciones en el uso de herramientas de IA para el proceso de formulación de políticas públicas son: National

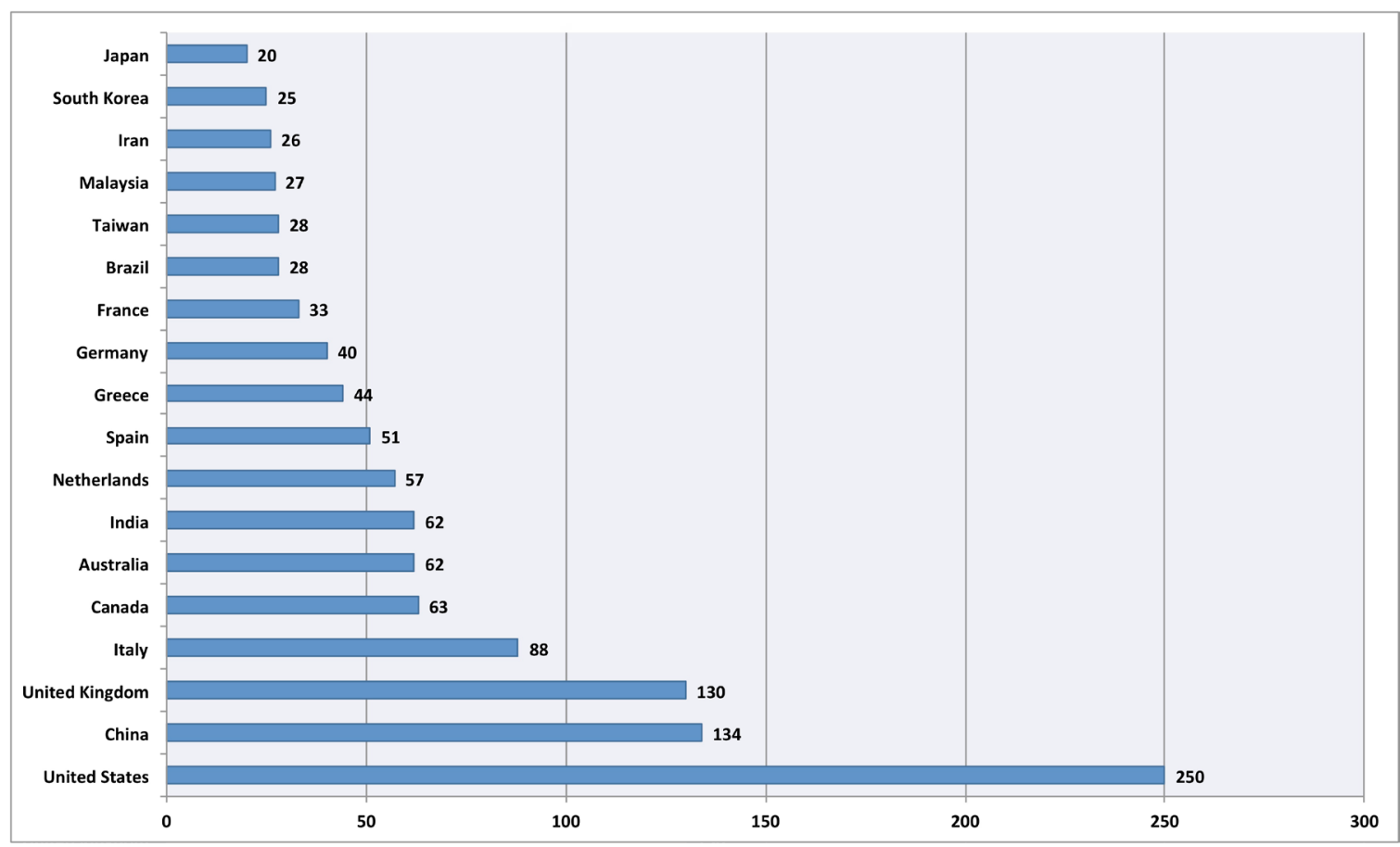

Figura 3. Publicaciones por países.

Fuente: adaptado por los autores de Scopus. 


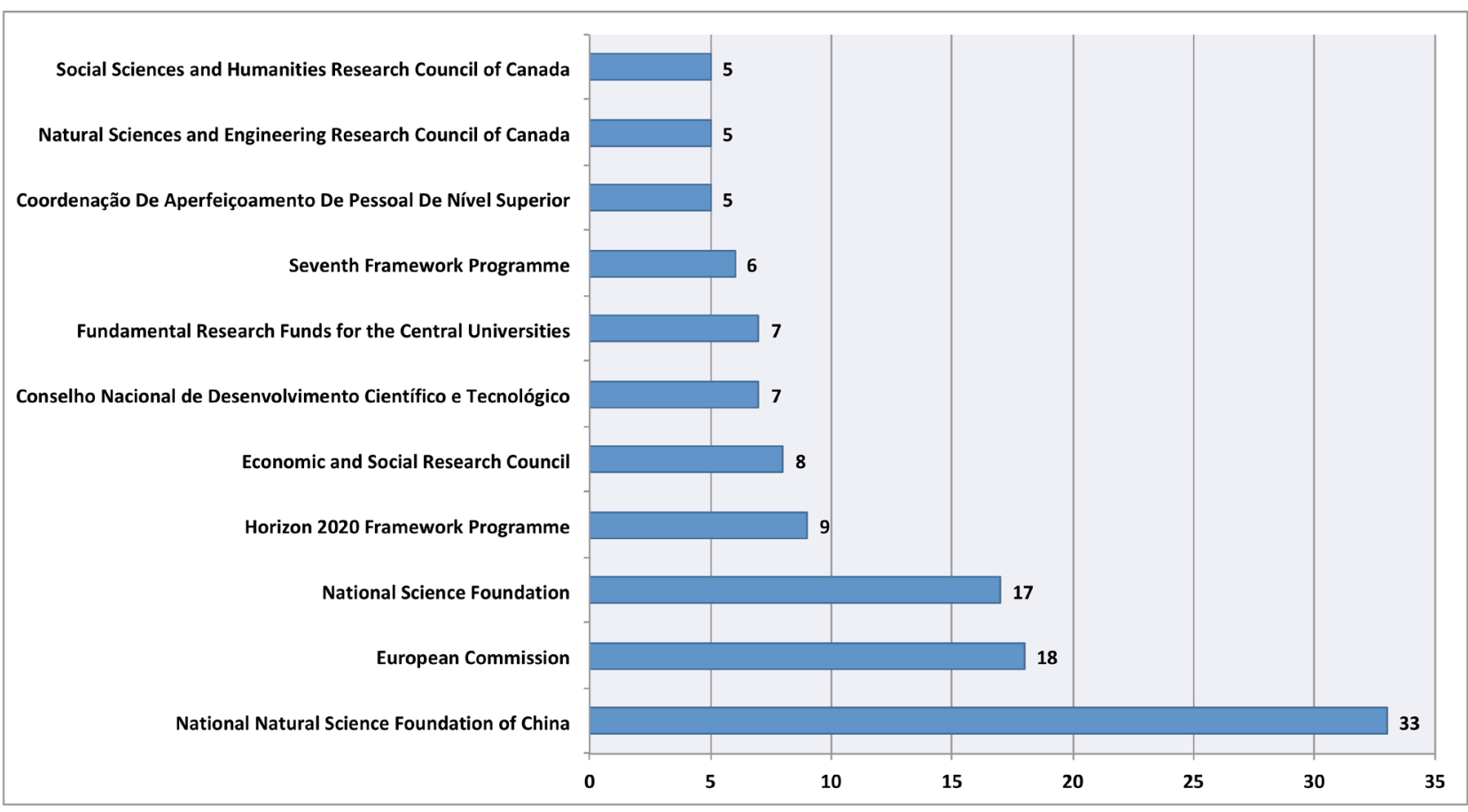

Figura 4. Patrocinadores.

Fuente: adaptado por los autores de Scopus.

Natural Science Foundation of China, European Commission y National Science Foundation. A continuación, se hace una breve reseña de estas.

- National Natural Science Foundation of China: fue creada en 1986 y estaba bajo la jurisdicción del Consejo de Estado; pero, a partir de 2018, se encuentra adscrito al Ministerio de Ciencia y Tecnología de la República Popular China. Esta organización tiene como misión apoyar la investigación, fomentar investigadores, desarrollar la cooperación internacional y promover el desarrollo socioeconómico (NSFC, 2020).

- -European Commission: tiene como objetivo desarrollar la estrategia general y la dirección política de la Unión Europea, para lo cual formula políticas para este fin y evalúa el desarroIlo de las mismas (Comisión Europea, 2020).

- National Science Foundation: la fundación es de carácter gubernamental, la cual financia la investigación y educación en campos de la ciencia y la ingeniería. Lo hace a través de subvenciones y acuerdos de cooperación con instituciones de educación, empresas, organizaciones de ciencias informales y otras organizaciones de investigación en Estados Unidos (National Science Foundation, 2020).

Como se observa, las tres principales organizaciones que apoyan la investigación en IA en política pública son de China, Estados Unidos y Europa; lo cual es coherente con lo analizado anteriormente. También es importante resaltar que las tres organizaciones son de carácter gubernamental, con lo cual se establece que es primordial el apoyo financiero del Estado para el desarrollo y avance científico.

\section{Palabras claves}

En la figura 5 se observa la red de relaciones de las palabras claves encontradas en las publicaciones. La herramienta informática que se usó fue VosViewer en su versión libre. 


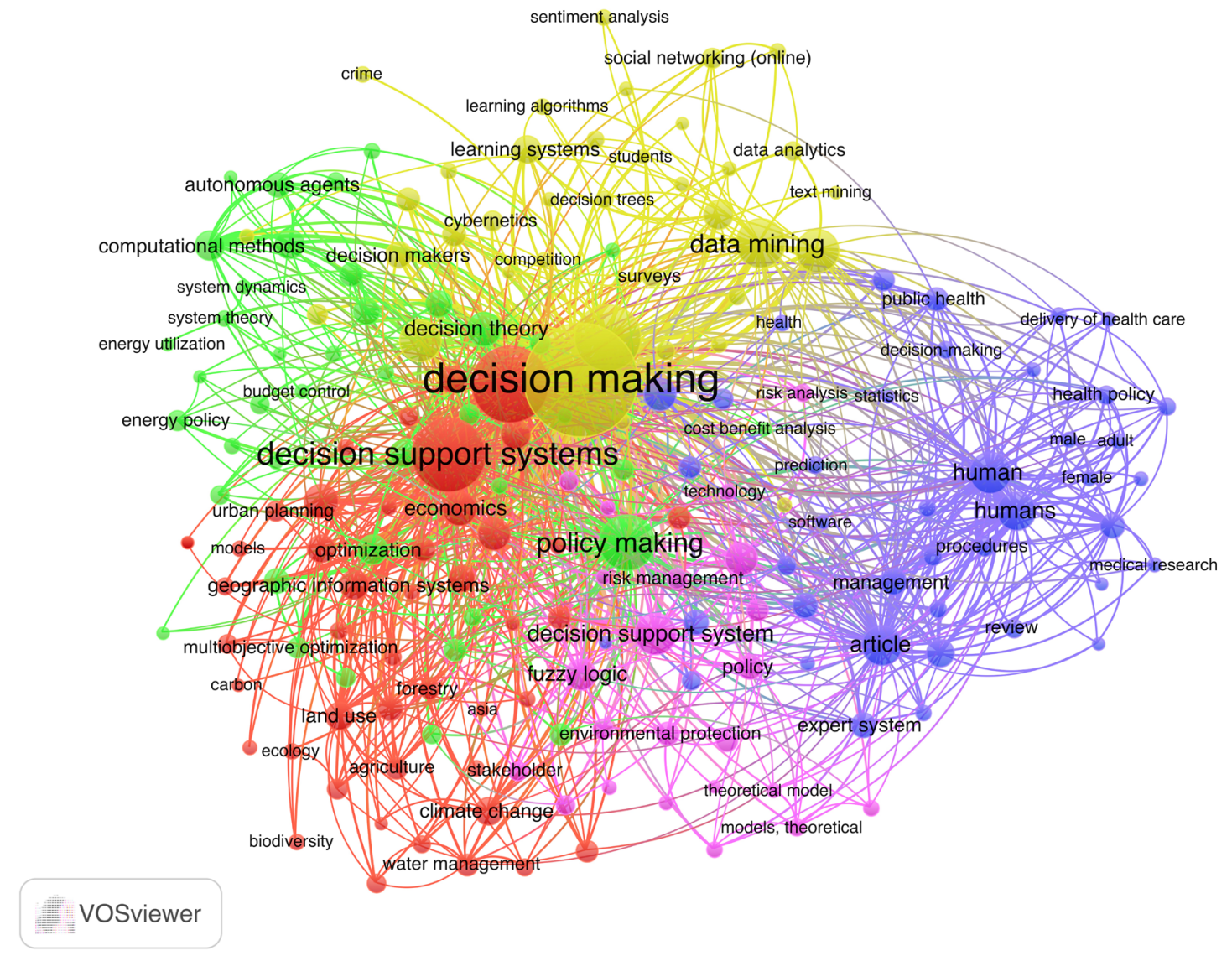

Figura 5. Publicaciones por países.

Fuente: adaptado por los autores de Scopus.

El software bibliométrico VosViever relacionó las palabras claves de las publicaciones y las juntó en cinco grupos, las cuales se distinguen en la figura 5 con diferentes colores. El primer grupo a nombrar es el correspondiente a las investigaciones desarrolladas para formular políticas públicas relacionadas con el área de la salud usando herramientas de IA. El segundo grupo corresponde al desarrollo de algoritmos para análisis de comportamientos en redes sociales paras así generar políticas públicas al respecto. El tercer grupo a la creación de modelos para generar políticas públicas en la generación y uso de energías. El cuarto grupo se relaciona con el uso de herramientas computacionales para formular políticas públicas en el sector agrícola, teniendo en cuenta el uso de la tierra, el agua y el cambio climático. Por último, el quinto grupo corresponde al desarrollo de sistemas de soporte de decisiones (DSS) para la generación de políticas ambientales. En resumen, el uso de herramientas de IA para la formulación de políticas públicas se ha usado principalmente en las áreas de la salud, energía, comportamientos sociales, agricultura y ciencias ambientales.

\section{Conclusiones}

Se evidencia la gran utilidad de las herramientas de IA para el proceso de formulación de políticas públicas; en especial las herramientas de big data, minería de datos y modelos basados en agentes.

En la última década se ha presentado un crecimiento en el interés científico en la aplicación de herramientas de IA en el proceso de formulación 
de políticas públicas. En el cual las herramientas preferidas para recopilación y procesamiento de información son la big data y minería de datos. El modelo basado en agentes es la herramienta que más se ha utilizado para pronosticar y evaluar los posibles resultados que se puedan obtener al implementar alguna política pública

Se observa el interés de los investigadores en desarrollar DSS que ayuden al proceso de formulación de políticas públicas, donde las herramientas de IA más utilizadas son minería de datos y modelos basados en agentes.

Los líderes en investigación y publicaciones en el uso de herramientas de IA aplicada al proceso de formulación de políticas públicas son Estados Unidos, República Popular de China y Reino Unido. Los otros países que realizan un número significativo de investigaciones sobre el tema son países desarrollados ubicados en Asia, Europa y Norte América. Lo cual se alinea con los principales patrocinadores de estas investigaciones que también corresponden a estos continentes. Estas organizaciones patrocinadoras son de carácter gubernamental, lo que evidencia la importancia del apoyo del gobierno para el desarrollo de estas investigaciones.

\section{Referencias}

Androutsopoulou, A., Charalabidis, Y. (2018). A Framework for Evidence Based Policy Making Combining Big Data, Dynamic Modelling and Machine Intelligence. ACM International Conference Proceeding Series, 575-583. https://doi.org/10.1145/3209415.3209427

Androutsopoulou, A., Karacapilidis, N., Loukis, E., Charalabidis, Y. (2018). Combining Technocrats" Expertise with Public Opinion through an Innovative e-Participation platform. IEEE Transactions on Emerging Topics in Computing, 6750(c), 1-14. https://doi.org/10.1109/TETC.2018.2824022

Androutsopoulou, A., Mureddu, F., Loukis, E., Charalabidis, Y. (2016). Passive Expert-Sourcing for Policy Making in the
European Union. IFIP International Federation for Information Processing, 9821, 55-66. https://doi.org/10.1007/978-3-319-45074-2

Baudasse, T., Calderón, C. (2009). Integración comercial del sector agrícola y desigualdad económica en los países en vías de desarrollo. Investigación Económica, 68(269), 37-72. https://doi.org/10.22201/ fe.01851667p.2009.269.16603

Borghesi, A., Milano, M. (2019). Merging observed and self-reported behaviour in agent-based simulation: A case study on photovoltaic adoption. Applied Sciences (Switzerland), 9(10). https://doi.org/10.3390/app9102098

Charalabidis, Y., Loukis, E. (2011). Transforming government agencies" approach to eparticipation through efficient exploitation of social media. 19th European Conference on Information Systems, ECIS.

Charalabidis, Y., Loukis, E., Androutsopoulou, A. (2011). Enhancing participative policy making through modelling and simulation: A state of the art review. Proceedings of the European, Mediterranean and Middle Eastern Conference on Information Systems - Informing Responsible Management: Sustainability in Emerging Economies, EMCIS, pp. 210-222.

Charalabidis, Y., Loukis, E., Spiliotopoulou, L., Diamantopoulou, V. (2013). A framework for utilizing Web 2.0 social media for participative governance. Proceedings of the European, Mediterranean and Middle Eastern Conference on Information Systems, EMCIS, pp. 0-15.

Chatzis, S. P., Siakoulis, V., Petropoulos, A., Stavroulakis, E., Vlachogiannakis, N. (2018). Forecasting stock market crisis events using deep and statistical machine learning techniques. Expert Systems with Applications, 112, 353-371. https://doi.org/10.1016/j.eswa.2018.06.032

Chávez, E. M., Arguello, A. M., Viscarra, C. P., Aro, G. L., Albarrasín, M.V. (2018). Inteligencia artificial en la toma de decisiones gerenciales. Revista Dilemas Contemporáneos: Educación, Política y Valores, 6(especial), 1-12. 
Chen, L., Qiao, Z., Wang, M., Wang, C., Du, R., Stanley, H.E. (2018). Which Artificial Intelligence Algorithm Better Predicts the Chinese Stock Market? IEEE Access, 6, 48625-48633. https://doi.org/10.1109/ ACCESS.2018.2859809

Chen, M., Hao, Y., Hwang, K., Wang, L., Wang, L. (2017). Disease Prediction by Machine Learning over Big Data from Healthcare Communities. IEEE Access, 5, 8869-8879. https://doi. org/10.1109/ACCESS.2017.2694446

Chen, P.T., Lin, C.L., Wu, W.N. (2020). Big data management in healthcare: Adoption challenges and implications. International Journal of Information Management, 102078. https://doi. org/10.1016/j.ijinfomgt.2020.102078

Cohen, M. D., March, J. G., Olsen, J. P. (1972). A Garbage Can Model of Organizational Choice. Administrative Science Quarterly, 17(1), 1-25. https://doi.org/10.2307/2392088

Comisión Europea (2020). Qué hace la Comisión Europea en materia de estrategia y políticas. $\underline{\text { ht- }}$ tps://ec.europa.eu/info/about-european-commission/what-european-commission-does/ strategy-and-policy es

Fernández, J. A., Martín, Q. M., Rodríguez, J. M. C. (2013). Business Intelligence Expert System on SOX Compliance over the Purchase Orders Creation Process. Intelligent Information Management, 5(3), 49-72. https://doi.org/http://dx.doi.org/10.4236/ iim.2013.53007

Flath, C. M., Stein, N. (2018). Towards a data science toolbox for industrial analytics applications. Computers in Industry, 94, 16-25. https:// doi.org/10.1016/j.compind.2017.09.003

Gavanelli, M., Milano, M., Bragaglia, S., Chesani, F., Marengo, E., Cagnoli, P. (2014). Multi-criteria optimal planning for energy policies in CLP? CEUR Workshop Proceedings, 1195, 54-68.

Gavanelli, M., Riguzzi, F., Milano, M., Cagnoli, P. (2016). Constraint and optimization techniques for supporting policy making. Computational
Intelligent Data Analysis for Sustainable Development, 361-381.

Gimona, A., Polhill, J. G. (2011). Exploring robustness of biodiversity policy with a coupled meta community and agent-based model. Journal of Land Use Science, 6(2-3), 175-193. https://doi. org/10.1080/1747423X.2011.558601

Gimona, A., Polhill, J.G., Davies, B. (2011). Sinks, sustainability, and conservation incentives. En J. Liu, V. Hull, A. T. Morzillo, J. A. Wiens (eds.), Sources, Sinks and Sustainability (pp. 155-178). https://doi.org/10.1017/ CBO9780511842399.010

Gómez, P. P. (2016). Evaluación de la política pública de reforma agraria en Colombia (19912010): Estudios de caso en seis municipios del país. Universidad Nacional de Colombia. http://www.bdigital.unal.edu.co/53481/

Huser, B., Rutledge, D. T., Van Delden, H., Wedderburn, M. E., Cameron, M., Elliott, S., ... Woods, R. A. (2009). Development of an integrated spatial decision support system (ISDSS) for local government in New Zealand. 18th World IMACS Congress and MODSIM09 International Congress on Modelling and Simulation: Interfacing Modelling and Simulation with Mathematical and Computational Sciences, Proceedings, 2370-2376.

Jiang, F., Jiang, Y., Zhi, H., Dong, Y., Li, H., Ma, S., ... Wang, Y. (2017). Artificial intelligence in healthcare: Past, present and future. Stroke and Vascular Neurology, 2(4), 230-243. https://doi.org/10.1136/svn-2017-000101

Lin, H., Zhu, H., Zuo, Y., Zhu, C., Wu, J., Xiong, H. (2017). Collaborative company profiling: Insights from an employee"s perspective. 31st AAAl Conference on Artificial Intelligence, AAAI, 1417-1423.

Loukis, E., Maragoudakis, M., Kyriakou, N. (2019). Economic Crisis Policy Analytics Based on Artificial Intelligence. IFIP International Federation for Information Processing 2019, 11685, 301-310. https://doi. org/10.1007/978-3-030-27325-5 
Manyika, J., Chui, M., Miremadi, M., Bughin, J., George, K., Willmott, K., ... Dewhurst, M. (2017). Un futuro que funciona: automatización, empleo y productividad. McKinsey Global Institute.

Marine-Roig, E. (2017). Measuring destination image through travel reviews in search engines. Sustainability (Switzerland), 9(8). https://doi. org/10.3390/su9081425

Mehta, N., Pandit, A. (2018). Concurrence of big data analytics and healthcare: A systematic review. International Journal of Medical Informatics, 114, 57-65. https://doi.org/10.1016/j. ijmedinf.2018.03.013

Menon, V. M., Rahulnath, H. A. (2017). A novel approach to evaluate and rank candidates in a recruitment process by estimating emotional intelligence through social media data. 2016 International Conference on Next Generation Intelligent Systems, ICNGIS. https://doi. org/10.1109/ICNGIS.2016.7854061

Milano, M. (2013a). Optimization for policy making: The cornerstone for an integrated approach. Lecture Notes in Computer Science (Including Subseries Lecture Notes in Artificial Intelligence and Lecture Notes in Bioinformatics), 8124 LNCS, 1-2. https://doi.org/10.1007/978-3-642-40627-0 1

Milano, M. (2013b). Sustainable energy policies: Research challenges and opportunities. Proceedings -Design, Automation and Test in Europe, DATE, 1143-1148. https://doi.org/10.7873/date.2013.239

Milano,M.,O"Sullivan, B., Gavanelli, M.(2014).Sustainable policy making: A strategic challenge for artificial intelligence. AlMagazine,35(3),22-35. https://doi.org/10.1609/aimag.v35i3.2534

Moon, S.H., Kim, Y.H., Lee, Y.H., Moon, B.R. (2019). Application of machine learning to an early warning system for very short-term heavy rainfall. Journal of Hydrology, 568, 1042-1054. https://doi.org/10.1016/j.jhydrol.2018.11.060

Morales, S.L., Morales, M.R., Rizo, R. (2017). Metodología para procesos de inteligencia de negocios con mejoras en la extracción y transformación de fuentes de Datos. Revista Publicando, 4(11), 107-119. http://rmlconsultores. com/revista/index.php/crv/article/view/553/ pdf 364

Moreno, J.M., Sánchez, J.M., Espítia, H.E. (2018). Water Level Prediction Using Artificial Neural Network Model. International Journal of Applied Engineering Research, 13(19), 1437814381.http://www.ripublication.com/ijaer18/ ijaerv13n19 45.pdf

National Science Foundation (2020). US NSFAbout Funding. https://www.nsf.gov/funding/ aboutfunding.jsp

Nawaz, N. (2019). Artificial intelligence interchange human intervention in the recruitment process in Indian software industry. International Journal of Advanced Trends in Computer Science and Engineering, 8(4), 1433-1442. https:// doi.org/10.30534/ijatcse/2019/62842019

Nayak, A., Pai, M.M.M., Pai, R.M. (2016). Prediction Models for Indian Stock Market. Procedia Computer Science, 89, 441-449. https://doi. org/10.1016/j.procs.2016.06.096

NSFC (2020). About Us-NSFC at a Glance. http://www.nsfc.gov.cn/english/site 1/about/6. $\underline{\mathrm{html}}$

Okesola, O.J., Okokpujie, K.O., Adewale, A. A., John, S.N., Omoruyi, O. (2018). An Improved Bank Credit Scoring Model: A Naïve Bayesian Approach. Proceedings - 2017 International Conference on Computational Science and Computational Intelligence, CSCI, 228-233. https://doi.org/10.1109/CSCI.2017.36

Ospina, D.M. (2017). Reivindicando al campesinado en Colombia: análisis de las fallas de redistribución y de reconocimiento en la implementación de las políticas agrarias de los siglos XX-XXI, y en la Política Pública de Víctimas y Restitución de Tierras. http://bdigital.unal. edu.co/64749/3/Trabajo $\% 20$ de $\% 20$ grado $\% 20$ Diana\%20Mendoza\%20Ospina\%202018.pdf

Peña, J.J., Chan, A.O., Balam, C. del C. (2017). Sistema experto en apoyo a toma de decisiones 
para aprobación de líneas de crédito. Pistas Educativas, 39(04), 402-411.

Polhill, J. G., Gimona, A. y Gotts, N. M. (2010). Analysis of incentive schemes for biodiversity using a coupled agent-based model of land use change and species metacommunity model. Modelling for Environment"s Sake: Proceedings of the 5th Biennial Conference of the International Environmental Modelling and Software Society, IEMS, 1, 809-816.

Polhill, J. G., Gimona, A., Gotts, N. M. (2013). Nonlinearities in biodiversity incentive schemes: A study using an integrated agentbased and metacommunity model. Environmental Modelling and Software, 45, 74-91. https://doi.org/10.1016/j.envsoft.2012.11.011

Poole, D., Mackworth, A. (2017). Artificial Intelligence: Foundations of Computational Agents. Cambridge University Press.

Ramesh, D., Suraj, P., Saini, L. (2016). Big data analytics in healthcare: A survey approach. International Conference on Microelectronics, Computing and Communication, MicroCom 2016. https://doi.org/10.1109/ MicroCom.2016.7522520

Russell, P., Norvig, S. (2009). Inteligencia Artificial, un enfoque moderno. Pearson.

Santacreu, L.J., Talavera, A., Aguasca, R., Galván, B.J. (2015). Sistema experto para tomar decisiones de emergencias y seguridad ante meteorología adversa. Dyna Ingenieria E Industria, 90(5), 502-512. https://doi.org/10.6036/7469

Šaur, D. (2017). Forecasting of Convective Precipitation Through NWP Models and Algorithm of Storms Prediction. Advances in Intelligent Systems and Computing, 573. https://doi.org/10.1007/978-3-319-57261-1

Shah, H., Tairan, N., Garg, H., Ghazali, R. (2018). A Quick Gbest Guided Artificial Bee Colony algorithm for stock market prices prediction. Symmetry, 10(7). https://doi.org/10.3390/sym10070292

Sibte, S., Abidi, R. (2001). Knowledge management in healthcare: towards. International Journal of Medical Informatics, 63, 5-18. https://doi. org/10.1016/S1386-5056(01)00167-8

Simon, H.A. (1979). Rational Decision Making in Business Organizations. The American Economic Review, 69(4), 493-513.

Soto, C. (2003). La agricultura comercial de los distritos de riego en México y su impacto en el desarrollo agrícola. Investigaciones Geográficas, Boletín Del Instituto de Geografía, Unam, 50, 173-195.

Thesmar, D., Sraer, D., Pinheiro, L., Dadson, N., Veliche, R., Greenberg, P. (2019). Combining the Power of Artificial Intelligence with the Richness of Healthcare Claims Data: Opportunities and Challenges.PharmacoEconomics, 37(6),745-752. https://doi.org/10.1007/s40273-019-00777-6

Turkson, R.E., Baagyere, E.Y., Wenya, G. E. (2016). A machine learning approach for predicting bank credit worthiness. 2016 3rd International Conference on Artificial Intelligence and Pattern Recognition, AIPR 2016, 81-87. https://doi.org/10.1109/ICAIPR.2016.7585216

Van Delden, H. (2009). Integration of socio-economic and bio-physical models to support sustainable development. 18th World IMACS Congress and MODSIM09 International Congress on Modelling and Simulation: Interfacing Modelling and Simulation with Mathematical and Computational Sciences, Proceedings, 2457-2463.

Van Delden, H., Hurkens, J. (2011). A generic integrated spatial decision support system for urban and regional planning. MODSIM 201119th International Congress on Modelling and Simulation-Sustaining Our Future: Understanding and Living with Uncertainty, 127-139. https://doi.org/10.36334/modsim.2011.keynote.vandelden

Van Delden, H., Kirkby, M.J., Hahn, B.M. (2009). Towards a modelling framework for integrated assessment in arid and semi-arid regions. 18th World IMACS Congress and MODSIM09 International Congress on Modelling and Simulation: Interfacing Modelling and Simulation 
with Mathematical and Computational Sciences, Proceedings, 3563-3569.

Van Delden, H., Seppelt, R., White, R., Jakeman, A. J. (2011). A methodology for the design and development of integrated models for policy support. Environmental Modelling and Software, 26(3), 266-279. https://doi. org/10.1016/j.envsoft.2010.03.021

Van Delden, H., Stuczynski, T., Ciaian, P., Paracchini, M. L., Hurkens, J., Lopatka, A., ... Vanhout, R. (2010). Integrated assessment of agricultural policies with dynamic land use change modelling. Ecological Modelling, 221(18), 2153-2166. https://doi.org/10.1016/j. ecolmodel.2010.03.023

Van Esch, P., Black, J. S., Ferolie, J. (2019). Marketing Al recruitment: The next phase in job application and selection. Computers in $\mathrm{Hu}$ man Behavior, 90, 215-222. https://doi. org/10.1016/j.chb.2018.09.009

Vargas, C., Téllez, G., Cubillos, A., Gómez, P., Garzón, L. (2016). Análisis de los beneficiarios de la política pública de reforma agraria en el marco del desarrollo rural en Colombia (1994-2010). Pampa, 13, 31-53. https://doi. org/10.14409/pampa.v0i13.5901

Zaidi, S. Z. H., Abidi, S. S. R., Manickam, S. (2002). Leveraging intelligent agents for knowledge discovery from heterogeneous healthcare data repositories. Studies in Health Technology and Informatics, 90, 335-340. https://doi.org/10.3233/978-1-60750-934-9-335

Zaidi, S. Z. H., Abidi, S. S. R., Manikam, S., Yu-N, C. (2004). ADMI: A multi-agent architecture to autonomously generate data mining services. 2004 2nd International IEEE Conference "Intelligent Systems" - Proceedings, 1, 273-279. https://doi.org/10.1109/is.2004.1344680

Zamora, A.P. (2017). Aplicación de la inteligencia artificial en la inversión de campañas publicitarias Application of artificial intelligent in the investment of advertising campaigns. Revista de Ciencia, Tecnología e Innovación, 4(3), 312-322. 